\title{
Pessoas idosas e tecnologias de informação e comunicação: inclusão digital como forma de inclusão social
}

\author{
Bárbara Barbosa Neves*
}

\section{Resumo}

É fundamental entender as razões de adoção ou não adoção das tecnologias de informação e comunicação pelas pessoas idosas, bem como o impacto do uso dessas tecnologias. Nesse intuito, revisito, nesta comunicação, uma pesquisa conduzida com pessoas idosas em Lisboa (Portugal) e descrevo os resultados preliminares de uma pesquisa em Toronto (Canadá) com idosos institucionalizados.

Palavras-chave: Info-exclusão. Exclusão social. Pessoas idosas. Internet. Literacia.

\section{Introdução}

As pessoas idosas (com 65 e mais anos de idade) continuam a ser o grupo etário que menos adota e utiliza as denominadas tecnologias de informação e comunicação (TIC), sobretudo a internet. De acordo com os últimos dados dos 28 países da União Europeia, 26\% de pessoas idosas (65-74 anos de idade) utilizam a internet enquanto que as pessoas entre os 16 e os 24 anos de idade atingem 88\%. (EUROSTAT, 2014). Em Portugal, $18,7 \%$ das pessoas idosas utiliza a internet (INE, 2013). No Brasil, em
$2013,11 \%$ das pessoas idosas com mais de 60 anos de idade usavam a internet, comparando com $77 \%$ dos Brasileiros entre os 16 e os 24 anos de idade (CGI, 2014). Embora a utilização da internet pelas pessoas idosas tenha aumentado nos últimos anos, há ainda uma visível diferença entre grupos etários. Assim, a idade tem sido um frequente preditor da info-exclusão ou do fosso digital (NORRIS, 2001; RICE; KATZ, 2003; CZAJA; LEE, 2007; MORRIS; GOODMAN; BRADING, 2007; NEVES; AMARO; FONSECA, 2013). A par desta info-exclusão, o discurso societário tende a percepcionar as pessoas idosas como tecnofóbicas, mas a literatura científica tem demonstrado que estas não se inserem tão facilmente nesse estereótipo (NEVES; AMARO, 2012). Portanto, esse discurso não só alimenta o idadismo (BUTLER, 1969), isto é a discriminação baseada na idade, como perpetua ideias que estigmatizam as pessoas idosas e que contribuem para uma visão unidimensional e homogénea deste grupo social.

O debate sobre a info-exclusão centra-se essencialmente naqueles que não têm acesso à internet, têm acesso limi-

\footnotetext{
" University of Melbourne, Parkville, Vitória, Austrália, barbara.barbosa@unimelb.edu.au
}

$\rightarrow$ http://dx.doi.org/10.5335/rbceh.v15i1.8787 
tado ou não têm a literacia digital para utilizar a internet (CASTELLS, 2001). A info-exclusão ultrapassa o mero acesso à internet ou às TIC e relaciona-se com um conjunto abrangente de desigualdades que passam por acesso e diferentes níveis de literacia. A investigação que tenho conduzido com pessoas idosas tem demonstrado que a info-exclusão deve ser também conceituada como forma de exclusão social.

Nesse contexto, a info-exclusão é uma forma de desigualdade social, quando impede o acesso das pessoas idosas a um conjunto de benefícios socioeconômi$\cos$, nomeadamente quando as excluí de:

- Possibilidades de comunicação potenciadas pelas TICs, nomeadamente pela internet. A internet permite comunicar de forma relativamente rápida e conveniente com uma variedade de laços sociais: laços fortes (familiares próximos, amigos próximos, etc.), laços fracos (conhecidos) e novos laços (pessoas que se conhecem online), sem os constrangimentos geográficos do passado (RAINIE; WELLMAN, 2012). Adicionalmente, a investigação sociológica mostra que a internet tem maioritariamente um efeito positivo na sociabilidade, interação social e sentido de comunidade e pertença social (RAINIE; WELLMAN, 2012; NEVES, 2013).

- Informação disponibilizada on-line, como informação sobre saúde e informação generalista e específica que pode permitir novas formas de aprendizagem ao longo da vida.
- Serviços on-line, como compras ou on-line banking, que podem facilitar o dia a dia das pessoas idosas e promover um envelhecimento ativo (RICE; KATZ, 2003; TORP et al., 2008; MITZNER et al., 2010; NEVES; AMARO, 2012).

- Acesso a serviços públicos, que cada vez mais migram para o formato digital, deixando de contemplar o formato físico. Por exemplo, a União Europeia dispõe de várias iniciativas de migração de serviços digitais, como o caso do "Digital by default" do governo britânico (GOV. UK, 2012).

- Participação social e cívica numa dimensão cada vez mais central da sociedade contemporânea.

Isso não quer dizer, no entanto, que só se contemplem vantagens na utilização das TIC pelas pessoas idosas. As TIC, como sistemas sociotécnicos, também implicam desvantagens, por exemplo, no foro da privacidade e da proteção pessoal de dados. Contudo, a aposta numa literacia crítica digital pode ajudar a ultrapassar algumas destas desvantagens (NEVES; AMARO, 2015).

\section{Adopção e não-adopção das TICs pelas pessoas idosas: um estudo em Lisboa}

Para se avaliar o nível de utilização das TICs (celulares, computadores e internet) pelas pessoas idosas (65+), tal como as suas atitudes e percepções sobre as novas tecnologias, conduzimos uma pesquisa em Lisboa com uma amostra 
aleatória estratificada de 500 pessoas idosas (2008-2010). Essa amostra, representativa da população idosa em Lisboa, respondeu a um inquérito por questionário. Esses dados quantitativos foram complementados com 10 entrevistas semiestruturadas, que permitiram aprofundar de forma qualitativa percepções e atitudes perante essas TICs. Os resultados deste estudo possibilitam examinar fatores para a adoção e não adoção das TIC e o seu impacto nesta população (NEVES; AMARO, 2012; NEVES; AMARO; FONSECA, 2013).

A amostra é composta por $60 \%$ de mulheres e $40 \%$ de homens, correspondendo a uma distribuição de género proporcional das pessoas idosas em Lisboa. A idade dos participantes é de 65 a 95 anos de idade $(\mathrm{M}=74,3, \mathrm{DP}=6,5)$. Cerca de metade dos inquiridos $(52 \%)$ são casados, $36 \%$ são viúvos, $7 \%$ são solteiros e $5 \%$ são divorciados. A maioria tem filhos (81\%) e netos (61\%). Em termos de habilitações literárias, $79 \%$ tem menos que educação secundária, $10 \%$ tem habilitações superiores, $6 \%$ não tem qualquer forma formal de educação e $3 \%$ tem educação secundária. Na sua globalidade, os participantes encontravam-se reformados (83\%).

Considerando os resultados descritivos da utilização das três TICs, $77 \%$ dos inquiridos tinham um telefone celular, $13 \%$ usavam um computador e $9 \%$ usavam a internet. A principal razão para utilizar essas TICs era para comunicar com familiares e amigos. Os telefones celulares também são utilizados para emergências $(22 \%)$, os computadores para escrever documentos de texto (24\%), e a internet para pesquisa (25\%) e lazer (13\%). As razões para não utilização dessas TICs são também semelhantes:

a) não ter necessidade: $34 \%$ no caso dos celulares, $24 \%$ no caso dos computadores e $44 \%$ no caso da internet;

b) não saber utilizar: $22 \%$ no caso dos celulares, $39 \%$ no caso dos computadores e $45 \%$ no caso da internet;

c) custo: $17 \%$ no caso dos celulares e $11 \%$ no caso dos computadores;

d) acesso: $12 \%$ no caso da internet.

Adicionalmente, $62 \%$ dos inquiridos concordava que as pessoas idosas deveriam aprender a utilizar computadores e a internet ( $22 \%$ discordava e $16 \%$ nem concordava nem discordava). Os dados qualitativos reforçam estes dados quantitativos, mas permitem contextualizar a adopção e não adopção dessas TICs. Dos dez entrevistados, oito tinham um celular, quatro usavam computadores e quatro usavam a internet. Os utilizadores de celulares associam-no com proximidade familiar, segurança, conveniência e com a diminuição de isolamento social. No entanto, todos à exceção de um, referem dificuldades visuais e físicas com os botões, com as mensagens de texto e com funções mais avançadas. Mesmo os não utilizadores reportam uma percepção positiva dos celulares, mencionando sobretudo a sua conveniência. No entanto, o custo é um obstáculo para a sua adoção. Os computadores são associados com conhecimento e lazer, enquanto a internet é associada com informação, serviços, interação social, proximidade familiar. 
A internet é a única dessas TICs que é relacionada com perigos: os entrevistados reportam os perigos que observam nesses meios, como fraude, pedofilia e pornografia infantil. Contudo, os utilizadores da internet indicam a vantagem de conseguir aceder a um manancial de informação on-line e de comunicar com familiares e amigos. Apenas um dos quatro entrevistados utilizava serviços on-line, como on-line banking. A não adoção de computadores e da internet prende-se com a falta de acesso e de literacia para o uso. Salienta-se a importância das relações intergeracionais para a adoção dessas TICs: todos os utilizadores enfatizaram que os netos foram os principais mentores da sua utilização de novas tecnologias.

Para analisar a significância de um conjunto de indicadores sociodemográficos (i.e., idade, sexo, educação, estado civil, ocupação, ocupação prévia, composição do agregado familiar e religião) na probabilidade de se usar celulares, computadores e internet nessa população, recorreu-se à regressão logística realizada com o método Forward: $L R$ no IBM SPSS 18. Esta análise revelou que idade e educação são os preditores dessas relações: por cada ano de idade a probabilidade de se usar essas tecnologias diminui e quanto mais elevado o nível de escolaridade, maior é a probabilidade de se usar tais tecnologias. As variáveis idade $\left(\mathrm{X}^{2} \operatorname{Wald}_{2}(1)=53,028, \mathrm{p}<0,001\right)$ e educação $\left(\mathrm{X}^{2}\right.$ Wald $(2)=13.768, \mathrm{p}=$ 0.001 ) apresentam um efeito estatisticamente significativo sobre a probabilidade de se usar celulares. Igualmente, Idade
$(\mathrm{X} 2$ Wald $(3)=13,030, \mathrm{p}=0,005)$ e educação $\left(X^{2}\right.$ Wald $\left.(3)=13,030, p=0,005\right)$ apresentam um efeito estatisticamente significativo sobre a probabilidade de se usar computadores. No entanto, apenas educação (X2Wald $(2)=7,166, p=0,028)$ tem um efeito significativo sobre a probabilidade de se usar internet. Assim, neste caso e ao contrário da literatura na área, a idade não é um preditor de utilização. Várias hipóteses para explicar esse resultado foram já avançadas em Neves, Amaro e Fonseca (2013), mas a conclusão principal é que fatores funcionais, como educação, literacia digital e acesso, são tão ou mais importantes do que fatores físicos, como a idade, para a adoção da internet.

No entanto, sempre que colocamos as lentes qualitativas para aprofundar temáticas verificamos que as categorias definidas são sempre mais fluídas do que esperado. Apesar dos esforços para delimitar analiticamente adoção e não adoção e utilização e não utilização, a riqueza da abordagem qualitativa revelou um conjunto de entrevistados que não se consideravam utilizadores, mas que utilizavam indiretamente a internet. Esses utilizadores indiretos usam a internet com a ajuda de outros para comunicar com familiares que vivem no estrangeiro (NEVES; AMARO, 2015). Mas, devido a baixos níveis de literacia, não conseguem utilizar a internet sozinhos necessitando de assistência contínua para o efeito. Assim, apesar de não se percepcionarem como utilizadores, atingem objetivos com o uso da tecnologia para interação social e tentam ultrapassar fatores funcionais 
(como a sua iliteracia digital) com fatores sociais (como apoio familiar). Esses resultados enfatizam a importância de se ultrapassar dicotomias e binários monolíticos quando se estuda adopção e não adopção de TIC entre pessoas idosas. De igual forma, mostra a relevância das TICs, sobretudo da internet, para a conectividade familiar e social.

\section{TIC para reduzir 0 isolamento social e a solidão? uma pesquisa em Toronto}

A segunda pesquisa relaciona-se com um aplicativo de comunicação para tablets, desenvolvido no "Technologies for Aging Gracefully Lab" (TAGlab) da Universidade de Toronto. Esse aplicativo foi desenvolvido para combater o isolamento social e a solidão entre as pessoas idosas. $\mathrm{O}$ isolamento social e a solidão ocorrem mais frequentemente nessa população, devido a um conjunto de fatores que vão de redes sociais reduzidas, características do curso de vida, menos capital social, a problemas de saúde, etc. (BCMH, 2004). Tem vindo a tornar-se um risco emergente na América do Norte, sobretudo para as pessoas idosas que estão institucionalizadas, sofrem de debilidades físicas e cognitivas e vivem sozinhas (CNSC, 2014; PRIETO-FLORES et al., 2011; VICTOR; SCAMBLER, 2009). A literatura científica mostra que 10 a $43 \%$ de pessoas idosas na América do Norte estão socialmente isoladas (NICHOLSON, 2012). Um estudo longitudinal de 1.604 pessoas idosas nos EUA indica que $43 \%$ destes indivíduos se sentem sozinhos (PERISSINOTTO; CENZER; COVINSKY, 2012). De acordo com um relatório de 2012, $24 \%$ das pessoas idosas Canadianas gostavam de ter participado em mais atividades sociais no ano transato (CNSC, 2014).

Embora os conceitos de isolamento social e solidão estejam relacionados e sejam, por vezes, utilizados como sinónimos, são diferentes. Numa perspectiva multidimensional, o isolamento social pode ser definido como a falta de qualidade e/ou quantidade de laços sociais, níveis baixos de participação em atividades sociais, solidão e falta de apoio social ou capital social (CORNWELL; WAITE, 2009; BAECKER et al., 2014). A solidão é definida como um sentimento subjetivo de isolamento, de não pertença, e de falta de companhia (PERISSINOTTO; CENZER; COVINSKY, 2012). O isolamento social pode levar a sentimentos de solidão, mas a solidão não depende diretamente do isolamento social: uma pessoa pode ter uma vasta rede social e sentir-se sozinho ou não se sentir sozinho embora tenha uma rede social pequena.

$\mathrm{O}$ isolamento social e a solidão têm efeitos negativos na saúde, bem-estar e participação ativa na sociedade das pessoas idosas (NICHOLSON, 2012; PERISSINOTTO; CENZER; COVINSKY, 2012). As pessoas idosas que sofrem de isolamento social e solidão têm maior probabilidade de sofrer de depressão, stress, debilidades cognitivas e funcionais, morbidez e mortalidade (CORNWELL; WAITE, 2009; STEPTOE et al., 2013). Esses riscos para a saúde são comparáveis aos riscos do tabagismo 
e da obesidade (CORNWELL; WAITE, 2009). Mas os custos do isolamento social e da solidão vão além das questões de saúde - as pessoas idosas que estão socialmente isoladas estão afastadas das suas comunidades e têm menos probabilidade de se envolverem civicamente e de se voluntariarem. Assim, perdem os efeitos positivos da participação pública e nós, as suas comunidades, perdemos as suas importantes contribuições societárias. Portanto, o isolamento social e a solidão representam um custo e um desafio para as pessoas idosas, famílias e instituições sociais.

Para intervir nesse problema e no seguimento de várias recomendações que indicam que a sua redução passa pelo aumento de oportunidades para interação social (FINDLAY, 2003; MASI et al., 2011), o TAGlab criou o InTouch (Figura 1). O InTouch é um aplicativo de comunicação desenhado e testado para pessoas idosas que estão institucionalizadas e/ou que vivem em ambientes associados com isolamento social e solidão (BAECKER et al., 2014). Viver em instituições é um dos principais fatores de isolamento social e solidão, na medida em que, as pessoas idosas institucionalizadas tendem a ter redes sociais pequenas e níveis baixos de interação com os seus laços sociais (PRIETO-FLORES et al., 2011; BCMH, 2004).
Figura 1 - Interface do InTouch

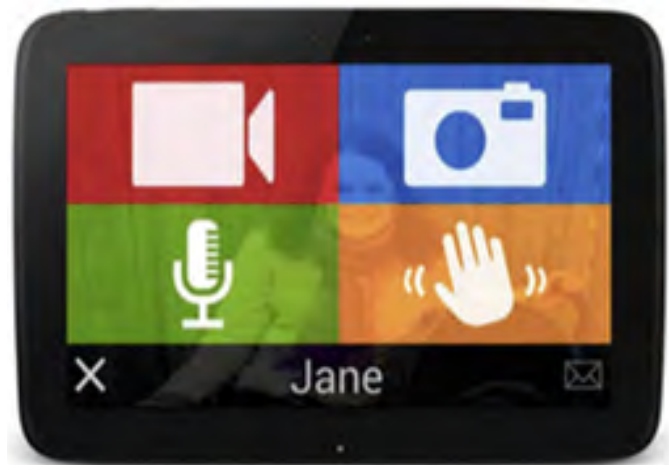

Nota: Opções quando se seleciona um contato: vídeo, foto, áudio e "wave" (mensagem pré-definida).

O InTouch é um aplicativo acessível que possui uma interface baseada em ícones (para que a sua utilização seja mais intuitiva e passível de uso em diferentes culturas) e que suporta comunicação assíncrona. $\mathrm{O}$ aplicativo foi desenvolvido para pessoas idosas com problemas motores (por exemplo, como resultado da doença de Parkinson) e, portanto, o utilizador não tem que datilografar ou teclar para o utilizar, apenas tocar. O aplicativo segue a metáfora do álbum de fotos: o tablet mostra várias fotografias de familiares e amigos e com um toque permite selecionar o familiar ou amigo que se pretende comunicar e enviar diferentes tipos de mensagens: uma mensagem pré-definida que diz "Estou a pensar em ti" (a "wave", isto é um 'aceno', representada por um mão), tal como mensagens de vídeo, áudio ou fotos. Familiares e amigos recebem essas mensagens nos seus e-mails, não necessitando de utilizar o InTouch para comunicar com o utilizador, e podem enviar para o InTouch vídeos, texto, áudio 
e imagens. Essa tecnologia é o resultado de vários protótipos e estudos de campo (BAECKER et al., 2014).

Para testar o InTouch e analisar fatores que facilitam e que dificultam a sua adoção, conduzimos duas pesquisas piloto em Toronto (2014). A primeira foi conduzida numa instituição de vida assistida com uma mulher idosa e a segunda foi conduzida numa instituição de longa permanência com cinco "pessoas idosas mais velhas", ou seja, com mais de 80 anos de idade. Desses participantes idosos mais velhos, dois eram homens e três eram mulheres; quatro sofriam de algumas debilidades físicas, nomeadamente três participantes eram sobreviventes de trombose e tinham um braço paralisado, estando numa cadeira de rodas, e uma participante sofria da doença de Parkinson. Dos seis participantes, apenas um tinha alguma literacia digital, pois sabia utilizar um computador e aceder à internet.

Esses dois estudos foram baseados em métodos mistos, tendo três fases: a primeira fase incluía uma sessão de treino individual com o participante e a aplicação de escalas de isolamento, solidão, depressão, interação social e apoio social. Após essa sessão, o InTouch era dado ao participante para usar livremente durante dois meses. Um mês depois dessa sessão, conduzimos um estudo de usabilidade e acessibilidade para testar como o participante utilizava o aplicativo e administramos as mesmas escalas. Dois meses depois da sessão inicial, conduzimos entrevistas semiestruturadas com os participantes e aplicamos a mesma bateria de escalas. Durante a pesquisa, visitamos os participantes uma vez por semana para recolher observações de campo.

A segunda pesquisa foi conduzida com chineses canadianos que não eram fluentes em Inglês, falando mandarim ou cantonês. A escolha desse grupo prendeu-se com a necessidade de testarmos um aplicativo baseado em ícones numa perspectiva multicultural. Contudo, apesar da importância da inclusão de diferentes grupos culturais, debatemo-nos com algumas dificuldades a nível do processo de investigação. Por exemplo, as escalas selecionadas para aferir interação social, isolamento social, solidão e apoio social são escalas validadas cientificamente em países ocidentais e encontravam-se descontextualizadas para os participantes chineses, uma vez que, estes tinham dificuldades em responder a alguns dos itens. Confrontados com essa dificuldade e porque não conseguimos encontrar uma bateria de escalas validadas em chinês, optamos por uma abordagem mais flexível durante a pesquisa. Adicionalmente, uma das participantes desse grupo teve que interromper o estudo após um mês, devido ao avanço de demência. $\mathrm{O}$ aplicativo deixou de ser usado e tornou-se impossível assegurar o seu consentimento informado. Por razões éticas e depois de consultar os profissionais geriátricos da instituição de longa permanência e familiares, optou-se por aconselhar a participante a desistir da pesquisa.

Os dados preliminares dessas pesquisas mostram que os principais fatores 
que facilitam a adoção desta tecnologia são sociais, atitudinais, físicos e de usabilidade. Dos cinco participantes, três eram mais ativos, usando o InTouch mais do que uma vez por semana, enquanto os restantes dois usavam o aplicativo uma vez por semana ou de quinze em quinze dias. Começando pelos fatores sociais, apoio social, ou seja, ter familiares envolvidos no processo, foi fundamental para a adopção do InTouch por parte de três participantes que nunca tinham tido qualquer contato com computadores ou internet anteriormente. Esse apoio social compensou, assim, a iliteracia digital dessas pessoas idosas. Esses três participantes tornaram-se os usuários mais frequentes do InTouch. Outros aspectos contextuais e culturais podem ainda facilitar ou dificultar a adopção de novas tecnologias de comunicação. Por exemplo, por motivos de segurança e porque os quartos dos residentes são partilhados (quatro camas por quarto) a instituição de longa permanência requeria uma forma de assegurar o tablet, o que implicou um cabo para ligar o dispositivo à cama dos participantes. Embora os participantes pudessem abrir o cabo para remover o tablet, isto requeria uma chave e devido à condição física da maioria impedia um uso mais livre e móvel do InTouch.

Idiossincrasias culturais podem também afetar a adoção e a utilização de um aplicativo. Por exemplo, um dos ícones, a "wave" (o 'aceno'), que representa uma mão a saudar e que envia uma mensagem pré-definida ("I am thinking of you"), não era percepcionada pelos participantes Chineses Canadianos como um sinal de saudação, mas como um sinal para cancelar uma ação. $O$ gesto da mão significa "não" na cultura Chinesa. $\mathrm{Na}$ nossa pesquisa anterior com protótipos do InTouch e com a participante piloto da instituição de vida assistida (participantes Canadianos, cuja língua mãe é o Inglês) a "wave" era considerada a função mais básica e, portanto, a opção mais utilizada pelos utilizadores principiantes. Contudo, como os participantes Chineses Canadianos não perceberam o significado cultural da "wave", apesar da sessão de treino e do manual, raramente utilizavam essa função.

Os fatores atitudinais incluem a percepção da utilidade do aplicativo e atitudes positivas de aprendizagem. Quatro dos cinco participantes reportaram que o aplicativo era útil para comunicar com familiares, sobretudo com os netos. A maioria dos participantes considerava que o InTouch os aproximava das novas gerações, que segundo eles eram as "gerações das tecnologias". A utilidade do aplicativo estava também relacionada com o facto de a tecnologia permitir diferentes modalidades de comunicação (fotos, texto, vídeo, áudio) e tipos de utilização: ativa ou passiva. Dois dos nossos utilizadores preferiam receber mensagens, enquanto os restantes gostavam da possibilidade de enviar e receber mensagens. Apesar de apenas um dos participantes ter alguma literacia digital, todos mostravam atitudes positivas relativamente à aprendizagem do InTouch. As sessões de treinos iniciais contemplaram uma abordagem de ensino que desconstruiu a ideia de "novas 
tecnologias", mostrando que um lápis e um fogão também são tecnologias. Essa abordagem, complementada em alguns casos com apoio familiar e noutros com o apoio dos investigadores, parece ter ajudado a reduzir dificuldades ou sentimentos de 'ansiedade' com as novas tecnologias.

Em relação aos fatores físicos, os quatro participantes mais velhos sofriam de alguma debilidade, o que limitava, por vezes, o seu uso do InTouch. Problemas motores e destreza eram os principais obstáculos. Nas palavras de uma das nossas participantes: "A minha mão não me ouve! As vezes a minha mão não me ouve..." Contudo, observamos também formas de compensar essas debilidades com outras estratégias, por exemplo, dois participantes que só tinham o uso de uma mão, apoiavam o tablet no corpo para facilitar a interação. Quanto aos fatores de usabilidade, a falta de familiaridade com convenções gestuais em dispositivos tácteis conduzia a alguma confusão entre tocar e arrastar elementos no écran. Alguns símbolos eram também entendidos de forma literal: o símbolo de mudança de direção da câmara para tirar fotos (duas setas em forma circular) era seguido com o dedo em vez de tocado por dois dos participantes.

A adopção de uma nova tecnologia de comunicação entre essa população resulta, assim, de uma complexa interação de fatores sociais, individuais e de usabilidade. Os dados preliminares demonstram que estes fatores não podem ser analisados isoladamente (por exemplo, apoio social compensa pela falta de literacia digital) tendo que ser adequadamente contextualizados.

No que diz respeito à utilização do InTouch para aumentar níveis de interação social e ajudar a reduzir isolamento social e solidão, estas pesquisas demonstram o potencial do InTouch para facilitar a comunicação entre pessoas idosas institucionalizadas e seus familiares. Todos os participantes indicaram que o InTouch os ajudava a estabelecer contato mais frequente com familiares e dos cinco, quatro sentiam-se mais incluídos na vida e na agenda familiar dos seus parentes. O participante que não reportou esse sentimento de inclusão familiar, era o participante que menos utilizava o InTouch, pois tinha apenas um familiar, o seu filho, que não estava muito envolvido no projeto. A percepção dos restantes participantes era de que agora podiam acompanhar mais frequentemente os seus familiares, sentindo maior proximidade familiar. Apesar desse aspeto positivo, encontramos diferentes expectativas e normas sociais em termos de comunicação entre familiares, que podem representar um obstáculo a uma interação mais prolongada. Em particular, havia alguma tensão nas relações intergeracionais, uma vez que, os netos enviavam mensagens e esperavam uma resposta imediata ou quase imediata dos avós. Os nossos participantes demoravam mais do que um dia ou uma semana a responder às mensagens, o que criava frustração para alguns familiares e os levou a reportar que o InTouch não era uma ferramenta viável de comunicação. Compreender 
essas diferentes expectativas, normas e padrões comunicacionais e integrá-los no desenvolvimento e na introdução ao sistema pode minimizar esse tipo de situações.

Concluindo, essas pesquisas mostram que esse aplicativo pode ajudar a aumentar a conexão social e a reduzir isolamento social e solidão entre idosos institucionalizados, mas que esse continua a ter um alcance limitado se não houver uma rede social disponível para aproveitar novas oportunidades de comunicação. Para continuar a aperfeiçoar o InTouch e a sua viabilidade como ferramenta de comunicação entre as pessoas idosas, encontramo-nos a preparar um estudo comparativo em vários tipos de instituições, incluindo pessoas idosas que vivem sozinhas, mas que são recipientes de serviços de apoio ao domicílio.

\section{Considerações finais}

Esses estudos de adoção de TICs entre pessoas idosas, embora em países diferentes e em grupos culturais distintos, chegam a resultados semelhantes. Em primeiro lugar, a relação entre TIC e pessoas idosas é mais complexa do que sugere o estereótipo da pessoa idosa excluída ou tecnofóbica. Encontramos diferentes tipos de utilizadores, não utilizadores e utilizadores indiretos, tal como atitudes globalmente positivas em relação às TICs.

Em segundo lugar, algumas das preconcepções em relação às TICs pelas pessoas idosas (i.e., dificuldade de utilização) são desconstruídas quando se considera uma abordagem de aprendizagem focada nas suas experiências, nos seus níveis de literacia digital e na sua familiaridade com outras tecnologias. Como refere Morris e colegas, até "a falta de interesse é causada por mal-entendidos sobre os computadores, que não são adequados para pessoas idosas, muito difíceis ou inúteis" (MORRIS; GOODMAN; BRADING, 2007, p. 49). Adicionalmente, só uma literacia digital crítica pode ajudar a ultrapassar potenciais efeitos negativos da utilização de TIC (como questões de privacidade e de proteção pessoal) e contribuir de forma sustentada para a integração de pessoas idosas nesta dimensão digital.

Em terceiro lugar, as TICs têm que ser conceituadas como sistemas socio-técnicos cujo desenvolvimento e adopção está dependente de contextos sociais específicos. Como é possível concluir da pesquisa preliminar com o InTouch, as novas tecnologias de comunicação podem facilitar interação e conexão social entre as pessoas idosas, mas continuam dependentes de enquadramentos sociais e relacionamentos interpessoais. $\mathrm{O}$ participante que menos usava o aplicativo era o que tinha apenas um familiar que não estava dedicado ao projeto. As novas tecnologias de comunicação podem combater isolamento social e solidão desde que adaptadas e integradas em conjunturas sociais, culturais, educacionais, fisiológicas, biopsicológicas e econômicas.

Em último lugar, quer a pesquisa em Portugal, quer no Canadá, sugere que inclusão digital pode ser uma forma de inclusão social. As pessoas idosas que 
usavam TIC reportavam acesso a serviços on-line, a fontes de informação mais variadas e a uma participação mais frequente na esfera familiar e numa esfera social e pública mais alargada; as pessoas idosas que usavam mais frequentemente o InTouch demonstravam indícios de uma crescente participação na esfera familiar. Aliás, num outro trabalho que realizei sobre capital social (i.e., recursos que estão disponíveis nos nossos laços sociais) e internet, os resultados mostram que os idosos que mais utilizavam a internet tinham maior probabilidade de terem níveis mais elevados de capital social (NEVES, 2013; NEVES; FONSECA, 2015). Contudo, a internet também reforça vantagem social acumulada e desigualdade social: como visto na pesquisa em Lisboa, a educação é um forte preditor de adoção da internet entre as pessoas idosas. Portanto, as pessoas idosas com habilitações literárias superiores parecem ter maior probabilidade de aceder aos benefícios sociais, psicológicos e económicos da internet, e manifestar um nível mais avançado de literacia para minimizar potenciais efeitos negativos da sua utilização.

\section{Older people and information and communication technologies: digital inclusion as a form of social inclusion}

\section{Abstract}

It's fundamental to understand the reasons for adoption and not adoption, as well as the impact of the use of information and communication technologies by the elder- ly. To illustrate this need and its importance, I review in this communication a survey conducted with elderly people in Lisbon (Portugal) and describe the preliminary results of a research in Toronto (Canada) with institutionalized elderly people.

Keywords: Info-exclusion. Social exclusion. Old people. Internet. Literacy.

\section{Referências}

BAECKER, R. et al. Technology to reduce social isolation and loneliness. In: Proceedings of the 16th international ACM SIGACCESS conference on Computers \& accessibility. ACM, p. 27-34, 2014.

BCMH. British Columbia Ministry of Health. Social isolation among seniors: an emerging issue. 2004. Disponível em: <https:// www.health.gov.bc.ca/library/publications/ year/2004/Social_Isolation_Among_Seniors. pdf> Acesso em: $1^{\circ}$ jan. 2014.

BUTLER, R. N. Age-ism: another form of bigotry. The Gerontologist, Oxford, v. 9, n. 4, p. 243-246, 1969.

CASTELLS, M. A galáxia internet: Reflexões sobre internet, negócios e sociedade. Lisboa: Fundação Calouste Gulbenkian, 2001.

CIG, 2014. TIC Domicílios e Empresas 2013. Pesquisa sobre o uso das tecnologias de informação e comunicação no Brasil. Comitê Gestor da internet no Brasil: São Paulo. Disponível em: <http://www.cgi.br/media/ docs/publicacoes/2/TIC_DOM_EMP_2013_livro_eletronico.pdf>. Acesso em: 16 jan. 2015.

CNSC. Canadian National Seniors Council. Report on the social isolation of seniors. 2014. Disponível em: <http://www.seniorscouncil. gc.ca/eng/research_publications/social_isolation/page00.shtml>. Acesso em: 4 jan 2015.

CORNWELL E. Y.; WAITE, L. J. Social disconnectedness, perceived isolation, and health among older adults. Journal of Health 
and Social Behavior, Thousand Oaks, v. 50, n. 1, p. 31-48, 2009.

CZAJA, S.; LEE, C. The impact of aging on access to technology. Universal Access in the Information Society (UAIS), Switzerland, v. 5, p. 341-349, 2007.

EUROSTAT. Individuals frequently using the internet. 2014. Disponível em: <http:// epp.eurostat.ec.europa.eu/tgm/refreshTableAction. do?tab $=$ table \&plugin $=0 \&$ pcode $=-$ tin $00092 \&$ language $=e n>$. Acesso em: 10 jun. 2014.

FINDLAY, R. A. Interventions to reduce social isolation amongst older people: where is the evidence? Ageing and Society, Cambridge, v. 23, n. 5, p. 647-658, 2003.

GOV.UK. Digital Service Standard. 2012. Disponível em: <https://www.gov.uk/service-manual/service-standard>. Acesso em: 4 fev. 2014.

INE. Instituto Nacional de Estatística. Utilização da internet em Portugal. 2013. Disponível em: <http://www.ine.pt/xportal/ xmain?xpid=INE\&xpgid=ine_destaques\&DESTAQUESdest_boui=152142241\&DESTAQUESmodo=2>. Acesso em: 10 fev. 2014.

MASI, C. M.; CHEN, H.-Y.; HAWKLEY, L. C.; CACIOPPO, J. T. A meta-analysis of interventions to reduce loneliness. Personality and Social Psychology Review, Washington, v. 15, n. 3, 219-266, 2011.

MITZNER, T. L et al.;. Older adults talk technology: technology usage and attitudes. Computers in Human Behavior, Amsterdã, v. 26, n. 6 , p. $1710-1721,2010$.

MORRIS, A.; GOODMAN, J.; BRADING, $H$. Internet use and non-use: views of older users. Universal Access in the Information Society, Heidelberg, v. 6, n. 1, p. 43-57, 2007.

NEVES, B. B. Social capital and internet use: the irrelevant, the bad, and the good. Sociology Compass, New Jersey, v. 7, n. 8, p. 599-611, 2013.
NEVES, B. B.; AMARO, F. A utilização da internet pelas pessoas idosas: uma perspectiva crítica. In: PASQUALOTTI, A.; GIL, H.; AMARO, F. (Org.). Tecnologias de informação no processo de envelhecimento humano. Passo Fundo: Ed. Universidade de Passo Fundo, 2015. p. 193-220.

NEVES, B. B.; AMARO, F. Too old for technology? How the elderly of Lisbon use and perceive ICT. The Journal of Community Informatics, Cidade do Cabo, v. 8, n. 1, 2012.

NEVES, B. B.; AMARO, F.; FONSECA, J. R. S. Coming of (Old) Age in the digital age: ict usage and non-usage among older adults. Sociological Research Online, Guildford, v. 18, n. 2, 2013.

NEVES, B. B.; FONSECA, J. R. S. Latent Class Models in action: Bridging social capital \& internet usage. Social Science Research, Amsterdã, v. 50, p. 15-30, 2015.

NICHOLSON, N. R. A review of social isolation: an important but underassessed condition in older adults. The Journal of Primary Prevention, Switzerland, v. 33, n. 2-3, p. 137-152, 2012.

NORRIS, P. Digital divide: civic engagement, information poverty, and the internet worldwide. Cambridge: Cambridge University Press, 2001.

PERISSINOTTO, C. M.; CENZER, S.; COVINSKY, K. E. Loneliness in older persons: A predictor of functional decline and death. Archives of Internal Medicine, Chicago, v. 172, n. 14, p. 1078-1083, 2012.

PRIETO-FLORES, M-E. et al. Factors associated with loneliness of noninstitutionalized and institutionalized older adults. Journal of Aging and Health, Thousand Oaks, v. 23, n. 1, p. 177-194, 2011.

RAINIE, L.; WELLMAN, B. Networked. Cambridge: MIT Press, 2012. 
RICE, R.; KATZ, J. Comparing internet and mobile phone usage: digital divides of usage, adoption, and dropouts. Telecommunications Policy, Amsterdã, v. 27, n. 8-9, p. 597-623, 2003.

STEPTOE, A. et al.; Social isolation, loneliness, and all-cause mortality in older men and women. Proceedings of the National Academy of Sciences, Washington, v. 110, n. 15, p. 5797-5801, 2013.

TORP, S. et al. A pilot study of how information and communication technology may contribute to health promotion among elderly spousal carers in Norway. Health and Social Care in the Community, New Jersey, v. 16, n. 1, p. 75-85, 2008.

VICTOR, C.; SCAMBLER, S. The social world of older people: Understanding loneliness and social isolation in later life. New York: McGraw-Hill International, 2009. 